\title{
Analysis of $\gamma$-Hydroxybutyrate in Human Urine by LC-MS/MS Method and Its Forensic Application
}

\author{
Luan Yujing, Wang Ruihua and Dong Ying \\ Institute of Forensic Science, Ministry of Public Security, China
}

\begin{abstract}
GHB ( $\gamma$-hydroxybutyrate) is becoming popular recreational drugs. As a result of its strong sedative and amnesiac effects, GHB has been implicated in a number of DFSA cases. The natural presence of GHB in the human body and its rapid elimination after ingestion make it difficult to detect and to evaluate its roles in suspected GHB-facilitated assaults. The paper describes an analytical method for the determination of GHB in urine using LC-MS/MS. Samples were acidified by ammonium chloride solution and extracted with ethyl acetate, and then the extracts were analysed by LC-MS/MS. The limit of detection was $0.05 \mu \mathrm{g} / \mathrm{mL}(\mathrm{S} / \mathrm{N}=$ 3). The intra- and inter-day precision was within $10.0 \%$ at three concentrations. The methods were found to be sensitive, accurate, rapid and suitable for the forensic toxicology to test GHB in real cases.
\end{abstract}

Key words: $\gamma$-Hydroxybutyrate (GHB), urine, LC/MS/MS, forensic application.

\section{Introduction}

GHB ( $\gamma$-hydroxybutyrate) is becoming one of the most popular recreational drugs. As a result of its strong sedative and amnesiac effects, GHB has been implicated in a number of DFSA cases [1, 2]. The natural presence of GHB in the human body and its rapid elimination after ingestion make it difficult to detect and to evaluate its roles in suspected GHB-facilitated assaults. The chemical structure of $\gamma$-hydroxybutyrate is in Fig. 1.

Methods of analysis of $\gamma$-hydroxybutyrate using rapid colorimetric screening test, GC-MS and HPLC-UV have had limited success, which does not give the information necessary to identify the drugs and may not be sensitive enough to determine GHB [3-11]. The authors therefore designed a specific and sensitive method using LC-MS/MS (liquid chromatography-tandem mass spectrometry). However, there are few reports on the determination of $\gamma$-hydroxybutyrate in human samples. The aim of our work is to identify $\gamma$-hydroxybutyrate in the

Corresponding author: Luan Yujing, doctor, associate professor, main research field: toxicological chemistry. human urine in DFSA (drug-facilitated sexual assault) case by LC-MS/MS.

\section{Case History}

In a case which happened on campus, three female students were stabbed by a strange man. Three victims all had symptoms of dizziness, nausea, weakness. One of the victims rushed to hospital and the urine sample was collected for toxicological analysis.

\section{Materials and Methods}

\subsection{Chemicals and Standards}

Chromatographic grade methanol and acetonitrile were purchased from Merck (Darmstadt, Germany), and $\gamma$-hydroxybutyrate standard was purchased from Lancaster Synthesis (England). Standard stock solution containing $1 \mathrm{mg} / \mathrm{mL}$ of $\gamma$-hydroxybutyrate was prepared in deionized water and stored at $-20^{\circ} \mathrm{C}$. Working standards were prepared from standard stock solution by appropriate sequential dilutions with deionized water and stored at $4{ }^{\circ} \mathrm{C}$. All the other chemicals used in the experiments were of analytical grade. 
<smiles>CC(O)CC(=O)O</smiles>

Fig. 1 GHB chemical structure.

\subsection{Extraction Procedure}

For qualitative analysis, $4 \mathrm{~mL}$ of acetonitrile was added to $1 \mathrm{~mL}$ of the samples. The mixture was shaken for 1 min with a vortex mixer and was centrifuged $\left(10,000 \mathrm{~g}, 10 \mathrm{~min}, 4{ }^{\circ} \mathrm{C}\right)$. A $10 \mu \mathrm{L}$ aliquot was used for the analysis. The extract was

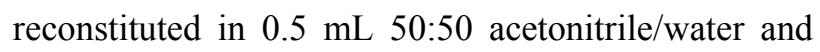
analysed.

\subsection{Instrumentation}

The analysis of $\gamma$-hydroxybutyrate was performed on Waters Xevo TQ MS (Waters, USA). Separation was performed with a Acquity UPLC BEH C18 column, $2.1 \times 50 \mathrm{~mm}$, packed with $1.7 \mu \mathrm{m}$ particles, which was maintained at $35{ }^{\circ} \mathrm{C}$. A gradient elution using water (with $0.1 \%$ formic acid of $10 \mathrm{mM}$ ammonium acetate, mobile phase A) and acetonitrile (aqueous mobile phase B) at a flow rate of $0.5 \mathrm{~mL} / \mathrm{min}$ was shown in Table 1 . Total run time was $4.8 \mathrm{~min}$.

Mass spectra were obtained using a Acquity UPLC ESI (system with electrospray ionization). The mass spectrometer was operated in MRM (multiple reaction monitoring) mode. Parameters were as follows: capillary voltage was set to $2.05 \mathrm{kv}$, desolvation gas to $500 \mathrm{~L} / \mathrm{Hr}$, cone gas to $50 \mathrm{~L} / \mathrm{Hr}$, desolvation temperature to $200{ }^{\circ} \mathrm{C}$ and ion source temperature to $150{ }^{\circ} \mathrm{C}$. Nitrogen, obtained from a nitrogen generator (99.93\%) was used for desolvation. MRM transitions of $\mathrm{m} / \mathrm{z} \quad 102.88 \rightarrow 56.97$ and $102.88 \rightarrow 84.90$ were monitored for $\gamma$-hydroxybutyrate at collision energy of $10 \mathrm{~V}$ and 15 $\mathrm{V}$, respectively.

\section{Results and Discussion}

The calibration curve was linear in the range of $1-1,000 \mu \mathrm{g} / \mathrm{mL}$ (too high concentration for LC-MS/MS) for $\gamma$-hydroxybutyrate, with correlation coefficients that were routinely greater than 0.998 in all cases. The detection limit of $\gamma$-hydroxybutyrate was $0.05 \mu \mathrm{g} / \mathrm{mL}(\mathrm{S} / \mathrm{N}=3)$. To establish the accuracy of the calibration curve, samples of low, medium and high concentrations of standard solutions were spiked with known amounts of $\gamma$-hydroxybutyrate and measured by the above method. The recovery was $86.2 \%-98.6 \%$. The intra- and inter-day precision was within $6.5 \%$ at three concentrations.

This study established an LC-MS/MS qualitative and quantitative analysis method for $\gamma$-hydroxybutyrate. The analysis time of this method is only $4.8 \mathrm{~min}$. Compared with the traditional methods, this method greatly shortens the analysis time and improves sensitivity. Since the procedure proved to be sensitive, selective and reproducible, the method developed was applied to the fatal case presented. In this case, the LC-MS/MS results showed the presence of $\gamma$-hydroxybutyrate in urine. The LC-MSMS chromatograms obtained from the extract of the victim's urine are shown in Fig. 2.

\section{Table 1 Gradient profile.}

\begin{tabular}{lll}
\hline Time & A $\%$ & B\% \\
\hline 0 & 2 & 98 \\
0.3 & 2 & 98 \\
2.0 & 50 & 50 \\
2.5 & 90 & 10 \\
4.0 & 2 & 98 \\
4.8 & 2 & 98 \\
\hline
\end{tabular}




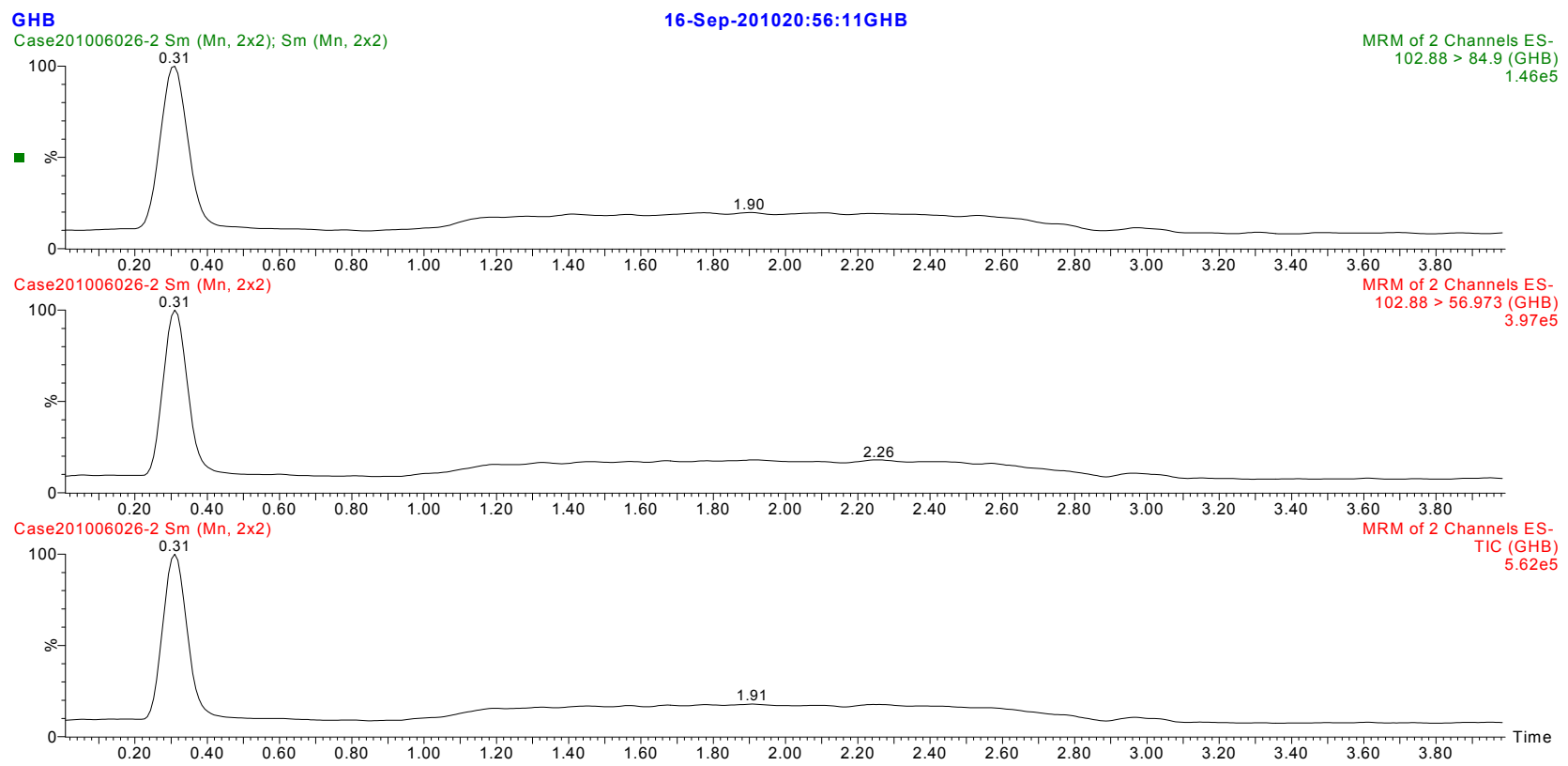

Fig. 2 LC-MS/MS chromatograms with two MRM transitions monitored in the victim's urine sample of the case report.

\section{Conclusions}

The LC-MS/MS method showed to be appropriate for screening, identification of $\gamma$-hydroxybutyrate in biological specimens. The extraction procedure is simple and effective. The method is suitable for the application to complex matrices like tissue samples. The low limit of detection enables the determination of $\gamma$-hydroxybutyrate even if there was a long period after poisoning.

\section{References}

[1] Lebeau, M., Andollo, M., and Hearn, W. L. 1999. "Recommendations for Toxicological Investigations of Drugs-facilitated Sexual Assaults.” J. Forensic Sci. 44: 227-30.

[2] Elsohly, M. A., and Salamone, S. J. 1999. "Prevalence of Drugs Used in Cases of Alleged Sexual Assault.” J. Anal Toxicol. 23: 141-6.

[3] Couper, F. J., and Logan, B. K. 2000. "Determination of $\gamma$-Hydroxybutyrate (GHB) in Biological Specimens by Gas Chromatography-Mass Spectrometry." J. Anal Toxicol. 24 (1): 1-7.

[4] McCusker, R. R., Wilkes, H. P., and Chronister, C. W. 1999. "Analysis of Gamma-Hydroxybutyrate (GHB) in Urine by Gas Chromatography-Mass Spectrometry." $J$. Anal Toxicol. 23: 301-5.

[5] Jiménez-Pérez, R., Sevilla, J. M., and Pineda, T. 2016. "Study of the Electro-oxidation of a Recreational Drug
GHB (Gamma Hydroxybutyric Acid) on a Platinum Catalyst-Type Electrode through Chronoamperometry and Spectro-Electrochemistry." $J$. of Electroanalytical Chemistry 766.

[6] William, C. A., and Karno, N. 2002. "Rapid Colorimetric Screening Test for $\gamma$-Hydroxybutyric Acid (Liquid X) in Human Urine." J. Forensic Sci. 126: 114-7.

[7] Blair, S., Song, M., Hall, B., and Brodbelt, J. 2001. "Determination of Gamma-Hydroxybutyrate in Water and Human Urine by Solid Phase Microextraction-Gas Chromatography/Quadrupole Ion Trap Spectrometry." J. Forensic Sci. 46: 688-93.

[8] LeBeau, M. A., Montgomery, M. A., and Miller, M. L. 2000. "Analysis of Biofluids for GHB (Gamma-Hydroxybutyrate) and GBL (Gamma-Butyrolactone) by headspace GC-FID and GC-MS.” J. Anal Toxicol. 24: 421-8.

[9] Mesmer, M. Z., and Satzger, R. D. 1998. "Determination of Gamma-hydroxybutyrate (GHB) and Gamma-Butyrolactone (GBL) by HPLC/UV-VIS Spectrophotometry and HPLC/Thermospray Mass Spectrometry." J. Forensic Sci. 43: 489-92.

[10] Anresen, H., Sprys, N., and Schmoldt, A. 2010. "Gamma-Hydroxybutyrate in Urine and Serum: Additional Data Supporting Current Cut-off Recommendations." Forensic. Sci. Int. 200 (1-3): 93-9.

[11] Bertol, E., Mari, F., Vaiano, F., Romano, G., Zaami, S., and Baglio, G. 2015. "Determination of GHB in Human Hair by HPLC-MS/MS: Development and Validation of a Method and Application to a Study Group and Three Possible Single Exposure Cases." Drug Test Anal 7 (5): 376-84. 